\title{
A NEW APPROACH TO TWO OVERDETERMINED EIGENVALUE PROBLEMS OF POMPEIU TYPE.
}

\author{
THIERRY CHATELAIN ${ }^{\dagger}$
}

\begin{abstract}
We are interested in two overdetermined problems in spectral theory, known as Schiffer's conjectures and related to the Pompeiu problem. We show the connection between these problems and the critical points of the eigenvalue functional with a volume constraint. In two dimensions, we use this fact to establish an integral identity satisfied by a conformal map.
\end{abstract}

\section{INTRODUCTION.}

In 1928, the rumanian mathematician Pompeiu introduced the notion of Pompeiu property for a domain :

$\Omega \subset \mathbb{R}^{N}$ is said to have the Pompeiu property if the only function $f \in C\left(\mathbb{R}^{N}\right)$ satisfying $\quad \int_{\sigma(\Omega)} f(x) d x=0 \quad$ for all rigid motions $\sigma$ of $\mathbb{R}^{N}$ is the zero function.

Then a natural question arises : what are the connected regular domains in $I R^{N}$ which have the Pompeiu Property?

Williams [14] has proved in 1976 that the Pompeiu Problem was equivalent to a conjecture of M. Schiffer in spectral theory :

let $\Omega$ be a connected regular domain in $\mathbb{R}^{N}$ such that there exists an eigenvalue $\mu$ and a Neumann-eigenfunction $u \neq 0$ satisfying

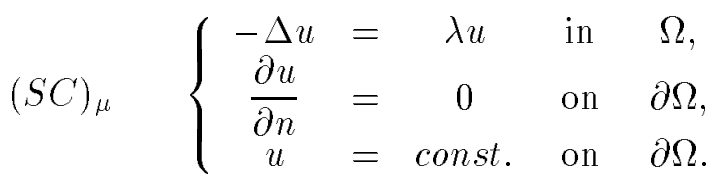

can we prove that $\Omega$ is a ball ?

This conjecture, for the Neumann case, can also, of course, be considered for the Dirichlet case : if $\lambda>0$ and $u \neq 0$ are such that

$$
(S C)_{\lambda}\left\{\begin{array}{ccccc}
-\Delta u & = & \lambda u & \text { in } & \Omega, \\
u & = & 0 & \text { on } & \partial \Omega, \\
\frac{\partial u}{\partial n} & = & \text { const. } & \text { on } & \partial \Omega .
\end{array}\right.
$$

then does it imply that $\Omega$ is a ball?

The motivation of this work is to give another interpretation of these two spectral overdetermined boundary value problems in two dimensions. First we prove that the solutions of these two problems $(S C)_{\lambda}$ and $(S C)_{\mu}$ are critical points of the eigenvalue functional under a volume constraint. Then we use this fact to obtain an interesting integral identity satisfied by some conformal map. 
In part 2, we recall the classical formulae of derivative of eigenvalues with respect to the domain. These formulae allow us to point out the link which exists between the Schiffer's conjecture $(S C)_{\lambda}$ and $(S C)_{\mu}$, and the critical points of the eigenvalue, considered as a domain functional, under a volume constraint. In this part we consider any single eigenvalue of the Laplacian operator.

In part 3, we restrict ourselves to the two-dimensional case. We consider for $\Omega$ a solution of $(S C)_{\lambda}$ or $(S C)_{\mu}$ and we introduce the conformal map $\phi$ which maps $\Omega$ onto the unit ball $D_{0}$. We map $\Omega$ into $\Omega_{t}$ thanks to a displacement field given by a perturbation $\psi$ of $\phi$ and we obtain, using the result of part 2 , the following integral identity satisfied by $\phi$ :

Theorem : $\exists c \in \mathbb{C}$ such that $\forall z \in D_{0}: c \phi^{\prime}(z)=\frac{1}{2 \pi} \int_{D_{0}} \frac{\phi^{\prime}(\eta) \hat{u}^{2}}{(1-\bar{\eta} z)^{2}} d \eta$ where $\hat{u}$ is the Dirichlet (or Neumann) eigenfunction for $(S C)_{\lambda}\left(\right.$ or $\left.(S C)_{\mu}\right)$ transferred to the unit disc.

\section{Critical points of the Eigenvalue Functional.}

\subsection{The Dirichlet Problem $(S C)_{\lambda}$}

Domain derivative is now a very usual tool in shape optimization. From a mathematical point of view, it goes back to Hadamard (1905) and Garabedian-Schiffer(1953). Good references are the works of Murat-Simon(1975) which are summed up in [11], or the more recent book of Sokolowski and Zolésio [13].

The $n^{\text {th }}$ eigenvalue of the Dirichlet-Laplacian, $\lambda_{n}(\Omega)$ which is characterized by

$$
l \lambda_{n}(\Omega)=\inf _{\substack{v \in W_{n}^{0} \\ v \neq 0}} \frac{\int_{\Omega}|\nabla v|^{2}}{\int_{\Omega} v^{2}}
$$

where $W_{n}^{0}=\left(\operatorname{Span}\left(u_{1}, \ldots, u_{n-1}\right)\right)^{\perp} \subset H_{0}^{1}(\Omega)$ and

$u_{1}, \ldots, u_{n-1}$ are the first $(n-1)$ eigenfunctions

can be regarded as a domain functional since it depends obviously on the domain $\Omega$.

The differentiability of $\lambda_{n}(\Omega)$ with respect to the domain $\Omega$ has been extensively studied by many authors, see e.g. [3], [4], [13], [1]. Let us recall the formula for a single eigenvalue in the next theorem.

Theorem 2.1. Assume that $\Omega$ is $C^{2}$ and that $V: \mathbb{R}^{N} \rightarrow \mathbb{R}^{N}$ is a $C^{2}$ deformation field that transforms $\Omega$ into $\Omega_{t}:=(I d+t V)(\Omega)$. Then the derivative of a single eigenvalue $\lambda_{n}(\Omega)$ with respect to the domain in the direction of the deformation field $V$ defined as

$$
\lambda_{n}^{\prime}(\Omega ; V):=d \lambda_{n}(\Omega ; V):=\lim _{t \rightarrow 0} \frac{1}{t}\left[\lambda_{n}\left(\Omega_{t}\right)-\lambda_{n}(\Omega)\right]
$$

exists and is given by

$$
d \lambda_{n}(\Omega ; V)=-\int_{\partial \Omega}\left(\frac{\partial u_{n}}{\partial \nu}\right)^{2} V \cdot \nu d \sigma
$$


where $\nu$ is the exterior normal vector to the boundary $\partial \Omega$, and $\frac{\partial u_{n}}{\partial \nu}$ is the normal derivative of the $n^{\text {th }}$ homogeneous Dirichlet-Laplace eigenfunction normalized by

$$
\int_{\Omega} u_{n}^{2}=1
$$

For a multi-valued eigenvalue we recall that the eigenvalue is no more Frechet differentiable, but we can give it's Clarke generalized gradient (see for instance [1], [4], [10] for more details).

Since the derivative of the volume is given by

$$
d V o l(\Omega ; V)=\int_{\partial \Omega} V . \nu d \sigma
$$

we have immediately, using relation (3) :

PROPOSITION 2.2. $\Omega$ is a solution of problem $(S C)_{\lambda}$ for a single eigenvalue $\lambda_{n}$ if and only if there exists a constant $c$ such that

$$
d \lambda(\Omega ; V)=-c^{2} d V o l(\Omega ; V) .
$$

In other words, the solutions of problem $(S C)_{\lambda}$ can be considered as critical points of the functional $\lambda_{n}(\Omega)$ with a volume constraint, the constant $-c^{2}$ being a Lagrange multiplier.

Proposition 2.2 is not new. It appears in a lecture of M. M. Schiffer in 1957 and in the more recent paper [4]. It has also been used in [2] or [8] to prove a similar symmetry result in the case of the first eigenvalue for inhomogeneous problems.

\subsection{The Neumann Problem $(S C)_{\mu}$}

For the homogeneous Neumann Problem, the characterization of $\mu_{n}(\Omega)$ is now

$$
l \mu_{n}(\Omega)=\inf _{\substack{v \in W_{n} \\ v \neq 0}} \frac{\int_{\mathbb{R}^{N}}|\nabla v|^{2}}{\int_{\mathbb{R}^{N}} v^{2}}
$$

where $W_{n}=\left(\operatorname{Span}\left(u_{0}, \ldots, u_{n-1}\right)\right)^{\perp} \subset H^{1}\left(\mathbb{R}^{N}\right)$ and $u_{0}, \ldots, u_{n-1}$ are the first $n$ eigenfunctions

and the derivative of a single eigenvalue $\mu_{n}(\Omega)$ with respect to the domain in the direction $V$ is given by

$$
d \mu_{n}(\Omega ; V)=-\int_{\partial \Omega}\left|\nabla u_{n}\right|^{2} V \cdot \nu d \sigma+\mu_{n}(\Omega) \int_{\partial \Omega} u_{n}^{2} V \cdot \nu d \sigma
$$

where $\nu$ is the exterior normal vector to the boundary $\partial \Omega$, and $u_{n}$ is the $n^{t h}$ homogeneous Neumann Laplace eigenfunction normalized by (4).

With formula (5) for the derivative of the volume we obtain now Proposition 2.3. $\Omega$ is a solution of problem $(S C)_{\mu}$ for a single eigenvalue $\mu_{n}$ if and only if there exists a constant $c$ such that

$$
d \mu(\Omega ; V)=-c^{2} d V o l(\Omega ; V) .
$$


So the solutions of problem $(S C)_{\mu}$ are also critical points of the functional $\mu_{n}(\Omega)$ with a volume constraint, the constant $-c^{2}$ being a Lagrange multiplier.

\section{AN INTEGRAL IDENTITY IN THE TWO-DIMENSIONAL CASE.}

We are now going to consider some particular deformations of the domain $\Omega$ induced by holomorphic maps. The main point is to obtain another expression of the derivative of the eigenvalue with respect to such deformations and then to apply the relation (6) or (9) in order to have some information on $\Omega$. Since the computations for the Neumann case are quite similar to the Dirichlet case, we restrict ourselves in the following to the Dirichlet case.

Let $\Omega \subset \mathbb{R}^{2}$ be a simply connected domain and let $\phi$ be the conformal map from the unit ball $D_{0}$ onto $\Omega$. Since classical regularity results for such free boundary problems imply that a solution $\Omega$ of $(S C)_{\lambda}$ or $(S C)_{\mu}$ in $I R^{2}$ must be analytic, we can assume without loss of generality that $\partial \Omega$ is analytic.

Let us fix, in all the following, a single Dirichlet eigenvalue, say $\lambda(\Omega)$, and let us denote by $u$ its eigenfunction normalized by (4). We define $\hat{u}$ on $D_{0}$ by :

$$
\hat{u}(\hat{z})=u(\phi(\hat{z}))
$$

Now, for all holomorphic function $\psi$, let us define a deformation of $\Omega$ by :

$$
\Omega_{t}=(\phi+t \psi)\left(D_{0}\right)=\left(I d+t \psi o \phi^{-1}\right)(\Omega) .
$$

We also define $u^{t}$ on $\Omega_{t}$ by transferring $\hat{u}$ to $\Omega_{t}$ :

$$
\hat{u}(\hat{z})=u^{t}((\phi+t \psi)(\hat{z})) \text {. }
$$

It is well-known that

$$
\int_{\Omega_{t}}\left|\nabla u^{t}(z)\right|^{2} d z=\int_{\Omega}|\nabla u(z)|^{2} d z
$$

and a simple change of variables yields

$$
\begin{aligned}
\int_{\Omega_{t}} u^{t}(z)^{2} d z & =\int_{D_{0}} \hat{u}(\hat{z})^{2}\left|\phi^{\prime}+t \psi^{\prime}(\hat{z})\right|^{2} d \hat{z} \\
& =\int_{D_{0}} \hat{u}(\hat{z})^{2}\left|\phi^{\prime}\right|^{2}+2 t \int_{D_{0}} \hat{u}(\hat{z})^{2} \operatorname{Re}\left(\phi^{\prime} \overline{\psi^{\prime}}\right)+O\left(t^{2}\right) \\
& =\int_{\Omega} u(z)^{2} d z+2 t \int_{D_{0}} \hat{u}(\hat{z})^{2} \operatorname{Re}\left(\phi^{\prime} \overline{\psi^{\prime}}\right)+O\left(t^{2}\right) .
\end{aligned}
$$

Now, (13) and (14) give :

$$
\frac{\int_{\Omega_{t}}\left|\nabla u^{t}(z)\right|^{2} d z}{\int_{\Omega_{t}} u^{t}(z)^{2} d z}=\lambda(\Omega)-2 t \lambda(\Omega) \int_{D_{0}} \hat{u}(\hat{z})^{2} \operatorname{Re}\left(\phi^{\prime} \overline{\psi^{\prime}}\right) d \hat{z}+O\left(t^{2}\right)
$$

and we have the following lemma :

Lemma 3.1. Let us denote by $u_{t}$ the (normalized) eigenfunction on $\Omega_{t}$ associated with $\lambda\left(\Omega_{t}\right)$. Then

$$
\frac{\int_{\Omega_{t}}\left|\nabla u^{t}(z)\right|^{2} d z}{\int_{\Omega_{t}} u^{t}(z)^{2} d z}=\frac{\int_{\Omega_{t}}\left|\nabla u_{t}(z)\right|^{2} d z}{\int_{\Omega_{t}} u_{t}(z)^{2} d z}+O\left(t^{2}\right) .
$$


Proof : Let us assume that $\lambda\left(\Omega_{t}\right)$ is the $n^{\text {th }}$ eigenvalue and let us denote by $u_{i t}, 1 \leq i \leq n-1$, the previous eigenfunctions on $\Omega_{t}$. We recall that $u^{t}$ is defined in (12) by transferring $\hat{u}$ to $\Omega_{t}$. We can write :

$$
\begin{aligned}
\left(u^{t}, u_{i t}\right) & =\left(u, u_{i}\right)+\left(u, u_{i t}-u_{i}\right)+\left(u^{t}-u, u_{i t}\right) \\
\left(u^{t}, u_{t}\right) & =(u, u)+\left(u, u_{t}-u\right)+\left(u^{t}-u, u_{t}\right)
\end{aligned}
$$

where (.,.) denotes the $L^{2}$ scalar product.

Since the variations of the domain $\Omega$ are regular, it follows from classical regularity results that $\nabla u_{i t}, \nabla u_{i}, u_{i t}$ and $u_{i}$ are uniformly bounded on $\overline{\Omega \cup \Omega_{t}}$.

Now, we use the differentiability of $t \mapsto u_{t}$ and $t \mapsto u^{t}$ together with the variational definition of the eigenfunctions to obtain :

$$
\left|\left(u^{t}, u_{t}\right)-(u, u)\right| \leq t M \quad \text { and } \quad\left|\left(u^{t}, u_{i t}\right)\right| \leq t M .
$$

So we can write

$$
u^{t}=\sum_{i=1}^{k-1} t \alpha_{i} u_{i t}+(1+\alpha t) u_{t}+o(t) .
$$

Then the lemma follows by a straightforward calculation using the orthogonality of the eigenfunctions.

Now, (15) and lemma 3.1 give :

$$
\lambda\left(\Omega_{t}\right)=\lambda(\Omega)-2 t \lambda(\Omega) \int_{D_{0}} \hat{u}(\hat{z})^{2} \operatorname{Re}\left(\phi^{\prime} \overline{\psi^{\prime}}\right) d \hat{z}+O\left(t^{2}\right)
$$

and passing to the limit in (16) as $t \rightarrow 0$, we obtain another expression of the derivative of $\lambda$ :

Proposition 3.2. The derivative with respect to the domain of $\lambda(\Omega)$ in the direction defined by the perturbation of the domain induced by $\psi$ (see (11)) is given by

$$
d \lambda(\Omega ; \psi)=-2 \lambda(\Omega) \int_{D_{0}} \hat{u}(\hat{z})^{2} \operatorname{Re}\left(\phi^{\prime} \overline{\psi^{\prime}}\right) d \hat{z}
$$

where $\hat{u}$ is given by $(10)$.

In the same way, we have :

Lemma 3.3. The derivative with respect to the domain of $V o l(\Omega)$ in the direction defined by the perturbation $\psi$ of $\phi$ is

$$
d \operatorname{Vol}(\Omega, \psi)=\frac{1}{2} \operatorname{Re} \int_{0}^{2 \pi} e^{i \theta}\left(\phi^{\prime} \bar{\psi}+\psi^{\prime} \bar{\phi}\right)\left(e^{i \theta}\right) d \theta .
$$

Proof : Stokes' formula gives

$$
\operatorname{Vol}\left(\Omega_{t}\right)=\frac{1}{2} \operatorname{Re} \int_{0}^{2 \pi} \mathrm{e}^{i \theta}\left(\phi^{\prime}+t \psi^{\prime}\right)\left(\mathrm{e}^{i \theta}\right)+(\overline{\phi+t \psi})\left(\mathrm{e}^{i \theta}\right) d \theta
$$

and using the definition of the derivative with respect to the domain we obtain the desired result.

Now, we can give our integral identity which is satisfied for $\Omega$ solution of problem $(S C)_{\lambda}$ : 
TheOrem 3.4. Let $\Omega$ be a solution of $(S C)_{\lambda}$ or $(S C)_{\mu}$ and let $\phi$ be the conformal map from the unit ball $D_{0}$ onto $\Omega$. Then :

$$
\exists c \in \mathbb{C} \text { such that } \forall z \in D_{0}: c \phi^{\prime}(z)=\frac{1}{2 \pi} \int_{D_{0}} \frac{\phi^{\prime}(\eta) \hat{u}^{2}}{(1-\bar{\eta} z)^{2}} d \eta
$$

where $\hat{u}$ is the eigenfunction transferred to the unit ball.

Proof : Using polar coordinates, we introduce :

$$
\begin{gathered}
\hat{z}=r e^{i \theta} \in D_{0} \\
\hat{u}^{2}\left(r e^{i \theta}\right)=\sum_{n=-\infty}^{n=+\infty} u_{n}(r) e^{i n \theta} \\
\phi(\hat{z})=\sum_{n=0}^{n=+\infty} a_{n} \hat{z}^{n} \\
\psi(\hat{z})=\sum_{n=0}^{n=+\infty} b_{n} \hat{z}^{n}
\end{gathered}
$$

and we have easily

$$
d V o l(\Omega ; \psi)=2 \pi \sum_{n=1}^{+\infty} n \operatorname{Re}\left(a_{n} \overline{b_{n}}\right)
$$

Now, using (18) - (21) and denoting $A_{j}=j a_{j}, B_{j}=j b_{j}$, we can write

$$
\begin{aligned}
\int_{D_{0}} \hat{u}^{2} \operatorname{Re}\left(\phi^{\prime} \overline{\psi^{\prime}}\right) & =2 \pi \operatorname{Re} \int_{0}^{1} \sum_{n=-\infty}^{+\infty} u_{n}(r) \sum_{k \geq M a x(0,-n)} A_{k+1} \overline{B_{k+n+1}} r^{2 k+n} d r \\
& =2 \pi \operatorname{Re} \int_{0}^{1} \sum_{N=0}^{+\infty}\left(\sum_{n=-\infty}^{N} u_{n}(r) A_{N-n+1} \overline{B_{N+1}} r^{2 N-n}\right) r d r \\
& =2 \pi \operatorname{Re} \int_{0}^{1} \sum_{N=1}^{+\infty} \overline{B_{N}} \sum_{n=-(N-1)}^{+\infty} u_{-n}(r) A_{N+n} r^{2 N+n-2} r d r \\
& =2 \pi \sum_{N=1}^{+\infty} \operatorname{Re}\left(\left[\sum_{k=1}^{+\infty} A_{k} \int_{0}^{1} u_{N-k}(r) r^{N+k-1} d r\right] \overline{B_{N}}\right)
\end{aligned}
$$

So, replacing in (17) we obtain

$$
d \lambda(\Omega ; \psi)=-4 \pi \lambda(\Omega) \sum_{n=1}^{+\infty} n \operatorname{Re}\left(\Gamma_{n} \overline{b_{n}}\right)
$$

with $\Gamma_{n}=\sum_{k=1}^{+\infty} k a_{k} \int_{0}^{1} u_{n-k}(r) r^{n+k-1} d r$.

Now, proposition 2.2 (or 2.3) implies

$$
\exists c \in \mathbb{C} \text { such that }\left(\Gamma_{n}\right)_{n \geq 1}=c\left(a_{n}\right)_{n \geq 1}
$$


(otherwise we can find some $\psi$ such that $d V o l(\Omega ; \psi)=0$ and $d \lambda(\Omega ; \psi) \neq 0)$.

By a further calculation we get

$$
\Gamma_{n}=\frac{1}{2 \pi} \int_{0}^{1} \int_{0}^{2 \pi} \phi^{\prime}\left(r e^{i \theta}\right) \hat{u}^{2}\left(r e^{i \theta}\right) r^{n-1} e^{-i(n-1) \theta} r d r d \theta
$$

Writing, for $\hat{z} \in D_{0}$

$$
c \phi^{\prime}(\hat{z})=\sum_{n=0}^{+\infty}(n+1)\left(c a_{n+1}\right) \hat{z}^{n}
$$

and using (24) we obtain our integral identity.

\section{CONCluding REMARKS}

Remark 1 : The two problems $(S C)_{\lambda}$ and $(S C)_{\mu}$ give the same integral identity (with different eigenfunctions $\hat{u}$ of course) of theorem 3.4. So if we were able to prove that this kind of integral identity be true only for $\phi=a z+b$ (i.e. only for a ball), we would have proved that the two problems have the same solution.

Remark 2 : According to Theorem 3.4, in order to solve the Schiffer's conjectures (and the Pompeiu problem) in two dimensions, a good strategy could consist in showing that the integral identity of theorem 3.4 is true only for $\phi$ a polynomial function. So we would be able to conclude thanks to the result of Garofalo-Segala [6].

\section{REFERENCES}

[1] Chatelain T., Choulli M., Clarke generalized gradient for eigenvalues, to appear in Communications in Applied Analysis.

[2] Chatelain T., Choulli M., Henrot A. , Some new ideas for a Schiffer's conjecture, Modelling and Optimization of Distributed Parameter Systems, Chapman and Hall, 1996.

[3] Chesnais D., Rousselet B., Continuité et différentiabilité d'éléments propres, application à l'optimisation de structures, Appl. Math. Optim., 22, 1990, p. 27-59.

[4] Cox S. J. , Extremal eigenvalue problems for the laplacian, Recent Advances in Partial Differential Equations, M. A. Herrero and E. Zuazua eds, RAM, John Wiley and Sons Masson, New York, 1994.

[5] Garabedian P., Schiffer M., Convexity of domain functionals, J. Analyse Math. 2 (1953), p. 281-369.

[6] Garofalo N., Segala F., New results on the Pompeiu problem, Trans. Amer. Math. Soc., 325 (1991), p. 273-286.

[7] Hadamard J. Mémoires sur un problème d'analyse relatif à l'équilibre des plaques élastiques encastrées. Oeuvres de J. Hadamard, Paris (1968).

[8] Henrot A., Philipppin G. A. , On a class of overdetermined eigenvalue problems, to appear in Math. Methods in the Applied Sciences.

[9] Pompeiu D. , Sur une propriété de fonctions continues dṕendant de plusieurs variables, Bull. Sci. Math. (2) 53, 328-332 (1929).

[10] Kato T. , Perturbation theory for linear operators, second edition, Springer-Verlag, Berlin, 1984.

[11] Simon J., Differentiation with respect to the domain in boundary value problems, Num. Funct. Anal. Optimz., 2 (7,8), 1980, p. 649-687.

[12] Schiffer M. M., Partial Differential Equations of the elliptic type, Lecture Series Symposium on Partial Differential Equations, Univ. of California, Berkeley, 1957, p. 97-149

[13] Sokolowski J., Zolésio J. P., Introduction to shape optimization: shape sensitity analysis, Springer Series in Computational Mathematics, Vol. 10, Springer, Berlin 1992. 
[14] Williams S. A., A partial solution of the Pompeiu problem, Math. Anal., 223 (1976), p. $183-190$.

†CNRS-Equipe de Mathématiques, Université de Franche-Comté, 16, route De Gray, 25030 Besançon, France 